\title{
Review of: "Effectiveness of a multidrug therapy consisting of Ivermectin, Azithromycin, Montelukast, and Acetylsalicylic acid to prevent hospitalization and death among ambulatory COVID-19 cases in Tlaxcala, Mexico"
}

Gonzalo Aranda-Abreu ${ }^{1}$

1 Universidad Veracruzana

Potential competing interests: The author(s) declared that no potential competing interests exist.

Interesting article on multidrug therapy against SARS-CoV-19 to prevent hospitalization. We particularly do not use ivermectin for treatment. However we do use azithromycin and acetylsalicylic acid. We also use amantadine as an anti-viral, where we have shown that also people with COVID-19 do not need to go to a hospital for mechanical ventilation.

The article is very interesting with good results. 